EGU21-9244

https://doi.org/10.5194/egusphere-egu21-9244

EGU General Assembly 2021

(c) Author(s) 2021. This work is distributed under

the Creative Commons Attribution 4.0 License.

\title{
Abel transform of exponential functions for planetary and cometary atmospheres with application to observation of 46P/Wirtanen and to the OI $557.7 \mathrm{~nm}$ emission at Mars.
}

\author{
Benoit Hubert ${ }^{1}$, Guy Munhoven ${ }^{1}$, Youssef Moulane ${ }^{2,3,4}$, Damien Hutsemekers ${ }^{2}$, Jean Manfroid ${ }^{2}$, \\ Cyrielle Opitom ${ }^{3}$, Emmanuel Jehin ${ }^{2}$, Shohei Aoki ${ }^{1,5}$, Lauriane Soret ${ }^{1}$, Leonardos Gkouvelis ${ }^{6}$, and Jean- \\ Claude Gérard ${ }^{1}$ \\ ${ }^{1}$ Université de Liège, Space sciences, Technologies \& Astrophysics Research (STAR) unit at the Institute of Astrophysics and \\ Geophysics, LPAP, Liège, Belgium \\ ${ }^{2}$ Université de Liège, Space sciences, Technologies \& Astrophysics Research (STAR) unit at the Institute of Astrophysics and \\ Geophysics, OrCa, Liège, Belgium \\ ${ }^{3}$ European Southern Observatory (ESO), Chile \\ ${ }^{4}$ Oukaimeden Observatory, High Energy Physics \& Astrophysics Laboratory, Cadi Ayyad University \\ ${ }^{5}$ Royal Belgian Institute for Space Aeronomy, Brussels, Belgium \\ ${ }^{6}$ AMES Research center, Moffett Field, Mountain view, CA, USA.
}

Line-of-sight integration of emissions from planetary and cometary atmospheres is the Abel transform of the emission rate, under the spherical symmetry assumption. Indefinite integrals constructed from the Abel transform integral are useful for implementing remote sensing data analysis methods, such as the numerical inverse Abel transform giving the volume emission rate compatible with the observation. We obtain analytical expressions based on a suitable, nonalternating, series development to compute those indefinite integrals. We establish expressions allowing absolute accuracy control of the convergence of these series depending on the number of terms involved. We compare the analytical method with numerical computation techniques, which are found to be sufficiently accurate as well. Inverse Abel transform fitting is then tested in order to establish that the expected emission rate profiles can be retrieved from the observation of both planetary and cometary atmospheres. We show that the method is robust, especially when Tikhonov regularization is included, although it must be carefully tuned when the observation varies across many orders of magnitude. A first application is conducted over observation of comet 46P/Wirtanen, showing some variability possibly attributable to an evolution of the contamination by dust and icy grains. A second application is considered to deduce the $557.7 \mathrm{~nm}$ volume emission rate profile of the metastable oxygen atom in the upper atmosphere of planet Mars. 